\title{
Confidence limit of the magnetotelluric phase sensitive skew
}

\author{
Pamela Lezaeta* \\ Freie Universität Berlin, FR Geophysik, Malteserstr. 74-100, 12249, Berlin, Germany
}

(Received January 3, 2001; Revised May 29, 2001; Accepted July 21, 2001)

\begin{abstract}
The rotationally invariant phase sensitive skew parameter, an indicator of dimensionality of conductivity structure, is a complicated non-linear function of the impedance tensor elements. In the presence of noise in the impedance data, skew can be significantly biased, leading to a false interpretation of dimensionality. Therefore, the probability function distribution of the skew parameter is derived to obtain its confidence limit, rather than treating a conventional linear propagation error. It is well known that the latter is only appropriate if the parameter is a function of independent random variables with small relative errors. The confidence limit is estimated by deriving its conditional probability function in terms of the tensor elements density function, using the Jacobi-matrix transformation of random variables, assuming the tensor elements to be normally distributed random variables. It is shown with synthetic and experimental data that the statistical confidence limit derived here truly reflects a probability range for the skew value. Bias of skew is seen to be significant with a small $5 \%$ of random Gaussian noise added to the tensor elements. Considering the $95 \%$ confidence limit instead of the measured skew itself results in a plausible approach to analyse dimensionality. The procedure developed here to estimate the confidence limit can also be extended to other functions of the tensor elements.
\end{abstract}

\section{Introduction}

When determining a parameter function of the impedance tensor, its corresponding error is normally not taken into account if it is a complicated non-linear function of the elements. One specific example treated here is the phase sensitive skew defined by Bahr (1991), which is a rotationally invariant parameter of the impedance tensor.

The skew parameter is based on the hypothesis that the impedance tensor is affected by telluric distortion, produced by shallow three-dimensional (3-D) anomalies overlaying a regional two-dimensional (2-D) structure (i.e., a superposition 2-D model). A 2-D model affected by telluric distortion implies equal phases between each pair of columm tensor elements (in the regional coordinate system). Skew measures these impedance phase differences and thus indicates the departure from two dimensionality. It would be zero if the telluric distortion hypothesis is valid for noisefree data, whereas values over 0.3 can be considered as an indicator of 3-D inductive effects (Bahr, 1991). However, with the addition of noise to the tensor elements the skew values can suffer significant bias, leading to a false interpretation of dimensionality. A way to avoid this problem is to estimate the probability function of skew, because the tensor elements with errors can be considered analogous to random variables. Thus, instead of the skew value itself, its probability threshold can provide a more plausible information on dimensionality.

In this paper, the confidence limits of the regional skew

*Now at: GFZ Potsdam, Telegrafenberg, 14473 Potsdam, Germany.

Copy right (C) The Society of Geomagnetism and Earth, Planetary and Space Sciences (SGEPSS); The Seismological Society of Japan; The Volcanological Society of Japan; The Geodetic Society of Japan; The Japanese Society for Planetary Sciences. are derived by expressing its distribution function in terms of the tensor element density functions. The Jacobi-matrix transformation of random variables is used in the derivation (e.g., Fisz, 1976). This procedure is valid for functions which are continuous and continuously differentiable. Skew fulfills these conditions. The transformation was considered by assuming the tensor elements as normally distributed random variables. The result is tested for synthetic data with random Gaussian noise added to the tensor. An example applied to measured data is also shown (Section 6). These experimental data were processed with a robust procedure (Egbert and Booker, 1986), which estimates an error covariance matrix for the impedance tensor, assumed to approach asymptotically a Gaussian distribution (Egbert, pers. comm.).

The scope of this paper is to show that the mathematical procedure performed in the derivation of the probability function distribution of the skew results in a feasible confidence limit to analyze dimensionality. Examples with synthetic and measured data demonstrates this. In a similar manner, the confidence limits of any other non-linear function of the tensor elements and hence the measured data can be estimated.

\section{The Variable Transformation for Skew}

Regional skew $(\eta)$ is a continuous function of the tensor elements. It has the following form:

$$
\eta=\frac{\sqrt{2\left|x_{1} x_{7}-x_{4} x_{6}+x_{2} x_{8}-x_{3} x_{5}\right|}}{\sqrt{\left(x_{2}-x_{3}\right)^{2}+\left(x_{6}-x_{7}\right)^{2}}}
$$

where, 


$$
\begin{array}{ll}
x_{1}=\operatorname{Re}\left\{Z_{x x}\right\} & x_{2}=\operatorname{Re}\left\{Z_{x y}\right\} \\
x_{3}=\operatorname{Re}\left\{Z_{y x}\right\} & x_{4}=\operatorname{Re}\left\{Z_{y y}\right\} \\
x_{5}=\operatorname{Im}\left\{Z_{x x}\right\} & x_{6}=\operatorname{Im}\left\{Z_{x y}\right\} \\
x_{7}=\operatorname{Im}\left\{Z_{y x}\right\} & x_{8}=\operatorname{Im}\left\{Z_{y y}\right\} .
\end{array}
$$

These variables correspond to the real and imaginary part of the impedance tensor $(\mathbf{Z})$ elements defined in magnetollurics:

$$
\mathbf{Z}=\left[\begin{array}{cc}
Z_{x x} & Z_{x y} \\
Z_{y x} & Z_{y y}
\end{array}\right]
$$

Skew $(\eta)$ is rotationally invariant and it vanishes if the response is equivalent to that from the ideal 2-D superposition model (i.e., electrostatic distortion without magnetic effect). This means that each pair $Z_{x x}, Z_{y x}$ and $Z_{x y}, Z_{y y}$ have equal phases.

Assuming a known density function distribution (d.f.) $f$ for the tensor elements $X=\left(x_{1}, \ldots, x_{8}\right)$ (Eq. (2)) assumed as random variables (r.v.) — we can derive the function distribution (f.d.) of $\eta$ in terms of $X$ by using the Jacobimatrix $J$ for the transformation of variables (e.g., Fisz, 1976).

The transformation of $X$ into $\eta$ is given by a space $Y$, which contains again the r.v.'s of $X$, except for one r.v. $x_{p}$ which is replaced by $\eta$ :

$$
\begin{aligned}
X & =\left(\ldots, x_{p-1}, x_{p}, x_{p+1}, \ldots\right) \rightarrow Y \\
& =\left(\ldots, x_{p-1}, \eta, x_{p+1}, \ldots\right) .
\end{aligned}
$$

The Jacobi transformation is valid if $\eta$ is continuously differentiable in $X$. This property is valid in the space of $X$ where the sum of variables contained in the modulus in the numerator of $\eta$ (Eq. (1)) is either a negative or a positive real number. Thus $\eta$ is continuously differentiable in $X$ except at $\eta(X)=0$, i.e., when the numerator of $\eta$ does not vanish. In order to fulfill this property for the further steps of the d.f. derivation, $\eta$ will be regarded statistically as a non-zero positive real number. The lowest limit of $\eta$ will be assigned as $0^{+}$. Note here that a zero skew value is only possible for noise-free data (for which no statistical error estimation of $\eta$ is required). Such data are only available from a numerical calculation of a perfect regional 2-D model.

It is also required for the derivation of the skew d.f. that $\eta$ with regards to the r.v. $x_{p}$ should satisfy the following conditions required by the Jacobi-transformation:

(1) $\eta$ is monotonic with respect to $x_{p}$, i.e., for a given $x_{p}^{a}<x_{p}^{b}$ in the range $(-\infty, \infty), \eta$ is either monotonically increasing if $\eta\left(x_{p}^{a}\right)<\eta\left(x_{p}^{b}\right)$, or monotonically decreasing if $\eta\left(x_{p}^{a}\right)>\eta\left(x_{p}^{b}\right)$.

(2) The partial derivative is equivalent with the inversion $\frac{\partial x_{p}}{\partial \eta}=\left(\frac{\partial \eta}{\partial x_{p}}\right)^{-1}$.

Condition (1) is not completely satisfied because $\eta$ is an absolute value as function of the tensor elements. A further analysis is required to account for this (Section 3 ). The second condition is true for the variables $x_{1}, x_{4}, x_{5}$ and $x_{8}$ having absolute partial derivatives of the form:

$$
\left|\frac{\partial x_{p}}{\partial \eta}\right|=\frac{\eta \cdot\left[\left(x_{2}-x_{3}\right)^{2}+\left(x_{6}-x_{7}\right)^{2}\right]}{\left|x_{i}\right|}
$$

for $(p, i)=(1,7),(4,6),(5,3),(8,2)$.

In consequence, the choice of one of these $x_{p}$ 's is arbitrary in the transformation due to the symmetry of $\left|\frac{\partial x_{p}}{\partial \eta}\right|$. Note that these variables are the diagonal impedance elements $Z_{x x}$, $Z_{y y}$, where $\eta$ encounters a local minimum in them and is symmetrical with respect to this minimum (Fig. 1).

The off-diagonal elements $Z_{x y}, Z_{y x}$ do not fulfill condition (2) and therefore $\eta$ is not symmetrical about a minimum and a maximum value in the off-diagonal elements (Fig. 2).

Having satisfied these conditions, the density function $g(\eta)$ of skew takes the form (e.g., Fisz, 1976):

$$
g(\eta)=|\operatorname{det}[J(X / Y)]| \cdot f(X) ; \quad X=\left(x_{1}, \ldots, x_{8}\right)
$$

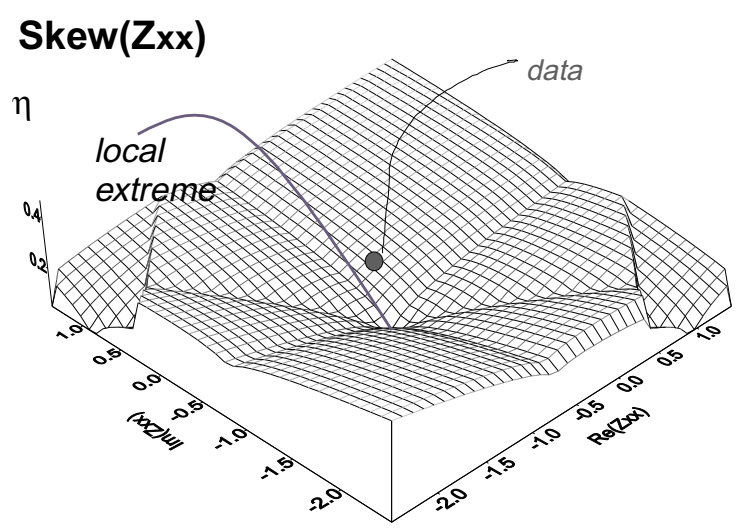

Fig. 1. Characteristic surface plot from synthetic data of the regional skew parameter $(\eta)$ as function of the real and imaginary part of the tensor element $Z_{x x}(\mathrm{~km} / \mathrm{s})$. The gridding interval of the variables is 0.01 centered on the extremal point, while the other tensor elements are kept fixed at their synthetic values. This plot is also characteristic for the other diagonal tensor element $Z_{y y}$. Any of these 4 diagonal elements can be used in the Jacobi-transformation of random variables.

\section{$\operatorname{Skew}(\mathrm{Zxy})$}

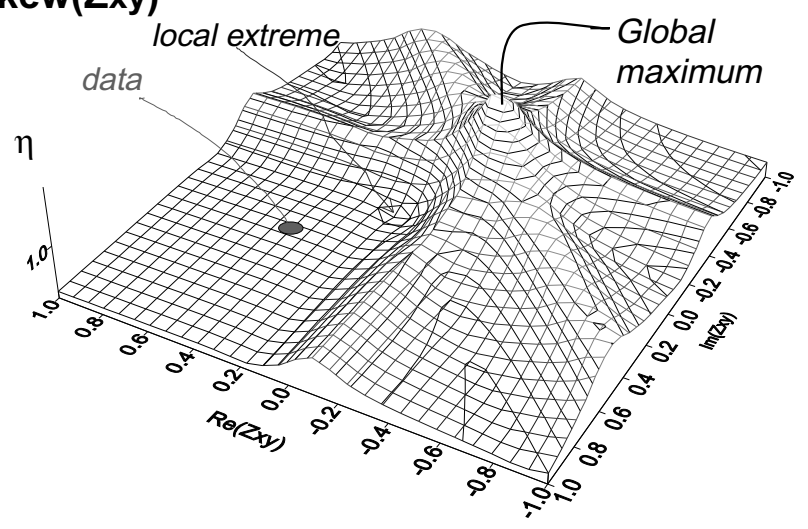

Fig. 2. Characteristic surface plot from synthetic data of the regional skew parameter $(\eta)$ as function of the real and imaginary part of the tensor element $Z_{x y}(\mathrm{~km} / \mathrm{s})$. Details as in Fig. 1. Skew has global positive and negative extreme. This is also true for the other off-diagonal tensor element $Z_{y x}$. These 4 elements cannot be used in the Jacobi-transformation of random variables (see text). 
The matrix $J$ is of dimension $8 \times 8$ determined by the number of r.v.'s contained in $X$, and have the partial derivatives of $X$ at $Y$ (Eq. (3)). $\operatorname{Det}[J(X / Y)]$ is its determinant $\left|\frac{\partial x_{p}}{\partial \eta}\right|$.

The expression for the multi-variate probability function of skew in terms of Eq. (5) is:

$$
\begin{aligned}
G(\eta)= & \int_{0^{+}}^{\eta_{o}} g(\eta) d \eta \\
= & \int_{0^{+}}^{\eta_{o}}\left(\int_{-\infty}^{\infty} \int_{-\infty}^{\infty} \ldots \int_{-\infty}^{\infty}\left|\frac{\partial x_{p}\left(\eta, \ldots, x_{p-1}, x_{p+1}, \ldots\right)}{\partial \eta}\right|\right. \\
& \left.\cdot f\left(\ldots, x_{p-1}, x_{p+1}, \ldots\right) \cdots d x_{p-1} d x_{p+1} \ldots\right) d \eta .
\end{aligned}
$$

Solving this multi-variate integration is complicated; to make further progress we simplify the problem to a univariate system. This implies determination of a conditional probability function for $\eta$ in terms of one r.v. $x_{p}$, while the other variables of $X$ are kept fixed at their respective mean values $u_{i}(i=1, \ldots, 8$ with $i \neq p)$. As mentioned above in regards to conditions 1 and 2 to validate the variable transformation, the r.v. $x_{p}$ should be one of the diagonal tensor elements.

\section{Derivation of the Probability Distribution of the Regional Skew Parameter}

The conditional probability function (p.f.) $P$ of $\eta\left(x_{p}\right)=$ $\eta_{p}$, given the known mean values $u_{i}$ of the tensor elements $(i \neq p)$, will be expressed as:

$$
G_{p}\left(\tilde{\eta}_{p}\right)=P\left(\eta_{p}<\tilde{\eta}_{p}\right)
$$

The derivation of $P$ should satisfy condition (1) of the variable transformation, i.e., $\eta$ should be either monotonically increasing or decreasing with respect to $x_{p}$. Also, the r.v. $x_{p}$ should be one of the diagonal tensor elements.

In order to simplify the following equations for the further derivation of $G_{p}$, the skew parameter from Eq. (1) will be expressed with the new term:

$$
\eta_{p}=\sqrt{\frac{2\left|x_{p}\left(s_{i} u_{i}\right)+c\right|}{d}}
$$

where the sub-index pairs $(p, i)$ are as in Eq. (4), and $d=$ $\left(u_{2}-u_{3}\right)^{2}+\left(u_{6}-u_{7}\right)^{2}$ is in the denominator of $\eta$ at the corresponding mean values. The parameter $c$ contains 6 conditional variables at their respective mean values and, $s_{i}= \pm 1$ corresponds to the sign of the respective pair $x_{p} x_{i}$ in Eq. (1).

Analysing for example the conditional probability for the r.v. $x_{1}$ (i.e., $\operatorname{Re}\left(Z_{x x}\right)$; Eq. (2)), then $u_{i}=u_{7}, s_{7}=1$ and $c=-u_{4} u_{6}+u_{2} u_{8}-u_{3} u_{5}$.

In the following, the p.f. $P$ of $\eta$ (Eq. (7)) is expressed in terms of Eq. (8), and after a change of variables a new expression is derived:

$$
G_{p}\left(\tilde{\eta}_{p}\right)=P\left(\sqrt{\frac{2\left|x_{p} s_{i} u_{i}+c\right|}{d}}<\tilde{\eta}_{p}\right)
$$

$$
\begin{aligned}
& =P\left(\left|x_{p} s_{i} u_{i}+c\right|<\frac{\tilde{\eta}_{p}^{2} d}{2}\right) \\
& =P\left(-\frac{\tilde{\eta}_{p}^{2} d}{2}<\left(x_{p} s_{i} u_{i}+c\right)<\frac{\tilde{\eta}_{p}^{2} d}{2}\right) .
\end{aligned}
$$

Assuming that the tensor elements are normally distributed, the r.v. $x_{p}$ has a normal d.f. $\phi\left(x_{p}\right)$ with mean $u_{p}$ and standard deviation $\sigma_{p}$.

The conditional p.f. $G_{p}(\eta)$ (Eq. (9)) for normally distributed tensor elements, expressed in terms of the standard distribution having a variance of 1 and mean $0\left(\psi_{o}\right)$ is:

$$
G_{p}(\eta)=\left\{\begin{array}{c}
\Psi_{o}\left(\frac{x_{p}^{+}(\eta)-u_{p}}{\sigma_{p}}\right)-\Psi_{o}\left(\frac{x_{p}^{-}(\eta)-u_{p}}{\sigma_{p}}\right) \\
\text { if } s_{i} u_{i}>0 \\
\Psi_{o}\left(\frac{x_{p}^{-}(\eta)-u_{p}}{\sigma_{p}}\right)-\Psi_{o}\left(\frac{x_{p}^{+}(\eta)-u_{p}}{\sigma_{p}}\right) \\
\text { if } s_{i} u_{i}<0
\end{array}\right.
$$

where the variables:

$$
\begin{aligned}
& x_{p}^{+}(\eta)=\frac{\eta^{2} d}{2 s_{i} u_{i}}-\frac{c}{s_{i} u_{i}} \\
& x_{p}^{-}(\eta)=\frac{-\eta^{2} d}{2 s_{i} u_{i}}-\frac{c}{s_{i} u_{i}} .
\end{aligned}
$$

The p.f. of $\eta$ (Eq. (10)) is related to the standardized folded normal distribution function (e.g., Dudewicz and Mishira, 1988). The two relations on the right comes from the monotonic condition for a valid transformation of spaces. The derivation of $G_{p}(\eta)$ is given in the appendix.

\section{Confidence Limit}

The confidence limit (C.L.) of the skew $(\eta)$ is defined as the probability $(P)$ that its true value $\eta_{o}$ has to lie within a certain range $\left[\eta_{a}, \eta_{b}\right]$. We use the conditional p.f. $G_{p}(\eta)$ of skew (Eq. (10)) to derive the confidence limit C.L., expressed as:

$$
P\left(\eta_{a}<\eta_{o}<\eta_{b}\right)=\text { C.L. }=G_{p}\left(\eta_{b}\right)-G_{p}\left(\eta_{a}\right) .
$$

Since the p.f. $G_{p}$ depends on the standardized normal distribution $\Psi_{o}$, which is symmetrical around the expected value, the desired confidence limit will be given by the following upper and lower limits:

$$
\begin{aligned}
G_{p}\left(\eta_{b}\right) & =\psi_{o}\left(\frac{x_{p}^{+}\left(\eta_{b}\right)-u_{p}}{\sigma_{p}}\right)-\psi_{o}\left(\frac{x_{p}^{-}\left(\eta_{b}\right)-u_{p}}{\sigma_{p}}\right) \\
& =\frac{1+\text { C.L. }}{2} \\
G_{p}\left(\eta_{a}\right) & =\psi_{o}\left(\frac{x_{p}^{+}\left(\eta_{a}\right)-u_{p}}{\sigma_{p}}\right)-\psi_{o}\left(\frac{x_{p}^{-}\left(\eta_{a}\right)-u_{p}}{\sigma_{p}}\right) \\
& =\frac{1-\text { C.L. }}{2}
\end{aligned}
$$

if $s_{i} u_{i}>0$, otherwise the indexes $a$ with $b$ and $b$ with $a$ should be exchanged, as indicated in Eq. (10). 
The confidence limit $\left(\eta_{a}, \eta_{b}\right)$ can be determined numerically with some iterative algorithm, since this cannot be solved directly by simply inverting the folded standard function $\Psi_{o}^{+}-\Psi_{o}^{-}$. The variables $\eta_{a}, \eta_{b}$ are the quantiles of the d.f. $G_{p}$ at the values $\frac{1-\text { C.L. }}{2}, \frac{1+\text { C.L. }}{2}$, respectively (provided that $s_{i} u_{i}>0$, otherwise the limits are reversed). Public function libraries written in Fortran as well as in C language can be used to calculate the quantile of a desired distribution function (e.g., Brandt, 1992). The algorithm to find C.L. consists of minimizing the function:

$$
\min \left\{\left(G_{p}\left(n_{a, b}^{j}\right)-\frac{1 \pm \text { C.L. }}{2}\right)\right\}
$$

with $\eta_{a, b}^{j}(j=0,1,2, \ldots)$ chosen iteratively in order to take appropriate values for the minimization function.

The result is dependent on the variable $x_{p}$ chosen, which can be either the element $R e Z_{x x}, \operatorname{Im} Z_{x x}, \operatorname{Re} Z_{y y}$ or $\operatorname{Im} Z_{y y}$. It is however advisable to select the variable which brings the largest confidence limit (Sections 5, 6).

\section{$4.195 \%$ confidence limit}

To show an example of the $95 \%$ confidence limit in terms of an explicit random variable $x_{p}$, consider this to be the element $x_{1}=\operatorname{Re} Z_{x x}$ (Eq. (2)), which, expressed in terms of $\eta$ (Eq. (1)) is:

$$
\begin{aligned}
x_{p}\left(\eta, x_{p-1}, \ldots\right)= & x_{1}\left(\eta, \hat{x}_{2}, \ldots, \hat{x}_{8}\right) \\
= & \frac{ \pm \eta^{2}}{2 \hat{x}_{7}} \cdot\left[\left(\hat{x}_{2}-\hat{x}_{3}\right)^{2}+\left(\hat{x}_{6}-\hat{x}_{7}\right)^{2}\right] \\
& +\frac{\left(\hat{x}_{4} \hat{x}_{6}-\hat{x}_{2} \hat{x}_{8}+\hat{x}_{3} \hat{x}_{5}\right)}{\hat{x}_{7}} .
\end{aligned}
$$

The variables of Eq. (11), derived from the transformation of limits of the probability function of $\eta\left(G_{p}\left(\eta_{p}\right)\right.$; Eq. (9)), are:

$$
x_{1}^{+}(\eta)=\frac{\eta^{2} d}{2 \hat{x}_{7}}-\frac{c}{\hat{x}_{7}}, \quad x_{1}^{-}(\eta)=\frac{-\eta^{2} d}{2 \hat{x}_{7}}-\frac{c}{\hat{x}_{7}}
$$

where

$$
\begin{aligned}
c & =-\hat{x}_{4} \hat{x}_{6}+\hat{x}_{2} \hat{x}_{8}-\hat{x}_{3} \hat{x}_{5} \\
d & =\left(\hat{x}_{2}-\hat{x}_{3}\right)^{2}+\left(\hat{x}_{6}-\hat{x}_{7}\right)^{2} \\
s_{i} & =s_{7}=1 \quad \text { and } \quad u_{i}=\hat{x}_{7} .
\end{aligned}
$$

Each $\hat{x}_{i}$ is the measured data considered as the mean value of the respective variable.

To derive the $95 \%$ confidence limit of $\eta$, i.e., C.L. $=0.95$, the function to minimize through successive iterations is:

$$
\min \left\{\left(G_{1}\left(n_{a, b}^{j}\right)-\frac{1 \pm 0.95 .}{2}\right)\right\} \quad \text { with } j=0,1,2, \ldots
$$

until the lower and upper limits $\eta_{a}, \eta_{b}$ are found for a given tolerance. $G_{1}(\eta)$ is the probability function of Eq. (10) at $p=1$, which approaches the lower and upper limits $\eta_{a}, \eta_{b}$ (or viceversa) as expressed in Eqs. (12).

\section{Example with Synthetic Data}

The confidence limit of $\eta$ was tested to the responses of a forward 3-D model, calculated with a modified version
(Mackie and Booker, 1999) of the algorithm developed by Mackie et al. (1994).

The model consists of a shallow 3-D conductive vertical dike (of $5 \Omega \mathrm{m}$ and $8 \mathrm{~km}$ depth) with horizontally finite extensions $\left(8 \times 40 \mathrm{~km}^{2}\right)$. It is embedded in a resistive medium $(500 \Omega \mathrm{m})$, and one of its edges is connected to a 2D conductive block $(1 \Omega \mathrm{m})$, which reaches a depth of $5 \mathrm{~km}$. The dike strikes by $45^{\circ}$ with respect to the conductive block. In Fig. 3, an horizontal view of the 3-D model at $2 \mathrm{~km}$ depth is presented. The skew values of two sites (ORI and CCO) were considered in the test. Site ORI is located near the centre of the dike and site $\mathrm{CCO}$ is above and near the edge of the dike (Fig. 3).

Gaussian noise was added to the tensor elements of the model responses with standard deviation of $2 \%$ and $5 \%$ of the largest tensor element amplitude. The procedure was repeated 100 times. The mean value of the random sample is the estimate of the noisy tensor element, and its error the estimate of the standard deviation.

The right hand plots of Figs. 4 and 5 show the 5\% noisy tensor elements with their errors, compared against the model responses. The skew parameters calculated from the model responses (ORI, CC0) and the noisy data (ran) are shown on the left hand plots of the figures. The $2 \%$ and $5 \%$ random noise data are illustrated separately with their respective $95 \%$ confidence limits. The latter comes from the conditional probability function of skew $\left(G_{p}(\eta)\right.$; Eq. (10)), which minimizes Eq. (13), for the variable $x_{p}$ (of one the diagonal tensor elements; Eq. (2)) resulting in the largest confidence limit, since this was seen to cover the region of the model response.

At site CC0 (located above the end of the dike; Fig. 3), the skew of the model response indicates that at the period range $100-500 \mathrm{~s}$ the departure from the 2-D superposition model is the highest, which means the most significant induction effect at these penetration depths. The noisy skew has been down biased indicating in contrast that the best fit with the 2$\mathrm{D}$ model hypothesis is at this period range. The confidence limit indeed reflects the range of the $95 \%$ probability in which the true skew value can lie.

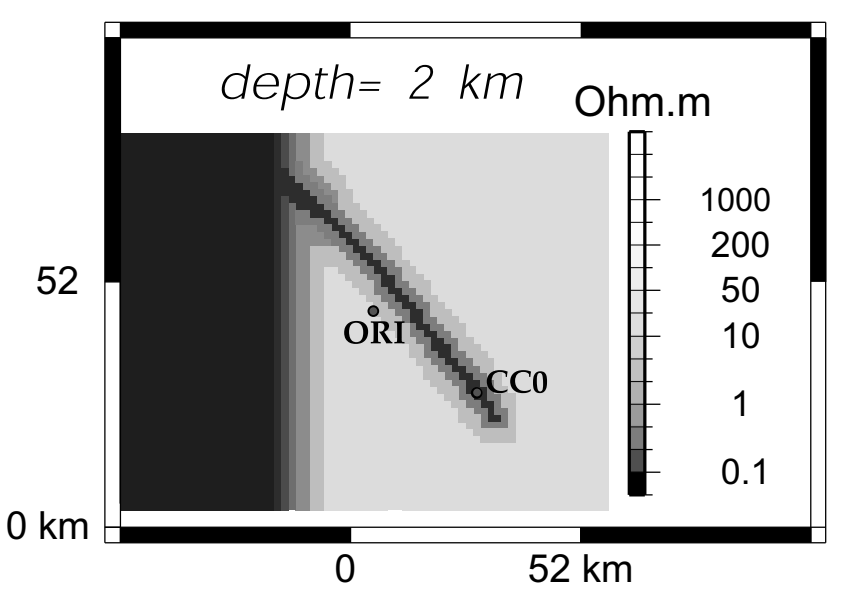

Fig. 3. Horizontal view of the 3-D model at $2 \mathrm{~km}$ depth. The mode responses at sites $\mathrm{ORI}$ and $\mathrm{CC} 0$, located next to the thin conductor, were used in the estimation of the skew confidence limits. 

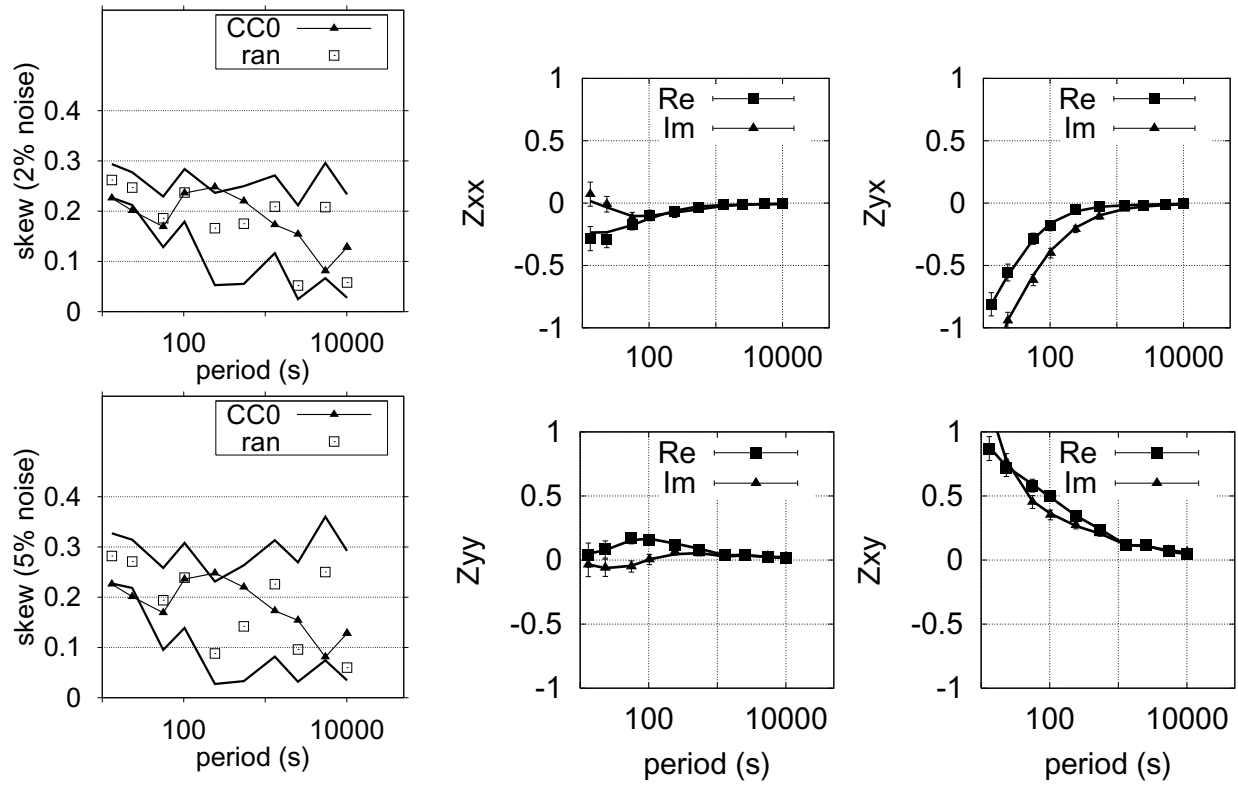

Fig. 4. An example of skew values using synthetic data (left), estimated from the tensor elements shown in right (units in km/s). The elements of the model response (lines) are shown over the data scattered with 5\% Gaussian noise (dots). The skew of the model response (CC0; in Fig. 3) is shown over the noisy skew (ran) within its $95 \%$ confidence limit. Above: Skew from the elements with $2 \%$ Gaussian noise. Below: Skew from the elements with $5 \%$ Gaussian noise.
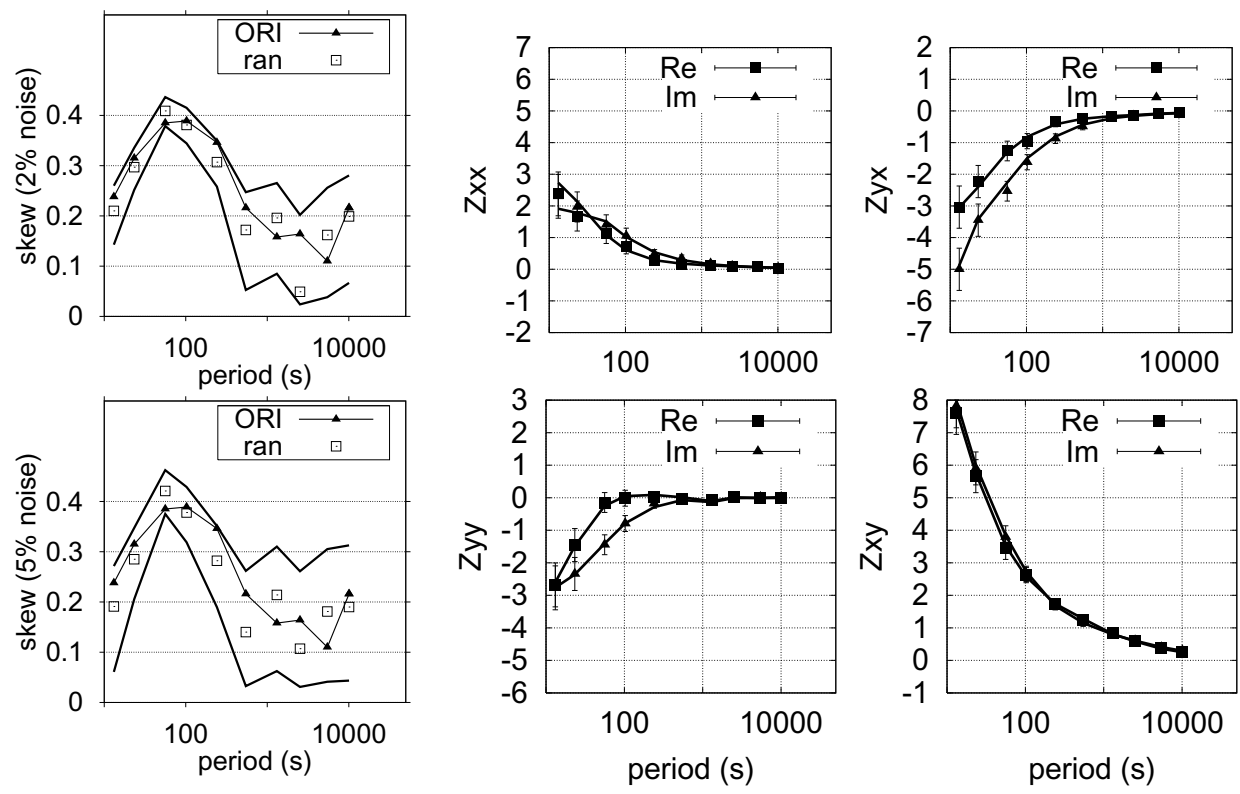

Fig. 5. As for Fig. 4, but with station ORI (from 3-D model; Fig. 3).

By site ORI (close to the centre of the dike; Fig. 3), the confidence limit of skew also reflects the $95 \%$ range probability of the true value. Their thresholds follow generally the trend of the real skew.

\section{Example with Field Data}

As an example, data from two stations (TIQ and GER) obtained from MT measurements carried out in the Southern Central Andes, within the framework of the German Collaborative Research Center "Deformation Processes in the Andes" (SFB 267, 2001), are shown. Time series data were processed using the robust technique of Egbert and Booker (1986), performing also a remote reference site to improve the data quality. The impedance tensor is estimated with an error covariance matrix, which is assumed to follow an asymptotically Gaussian distribution. This assumption of course improves with increasing number of sample data recorded.

Figure 6 shows the tensor elements of sites TIQ and GER, and the skew values with their confidence limits. The latter was estimated for the diagonal element variable which gave the largest confidence limit, analogous to the synthetic data 

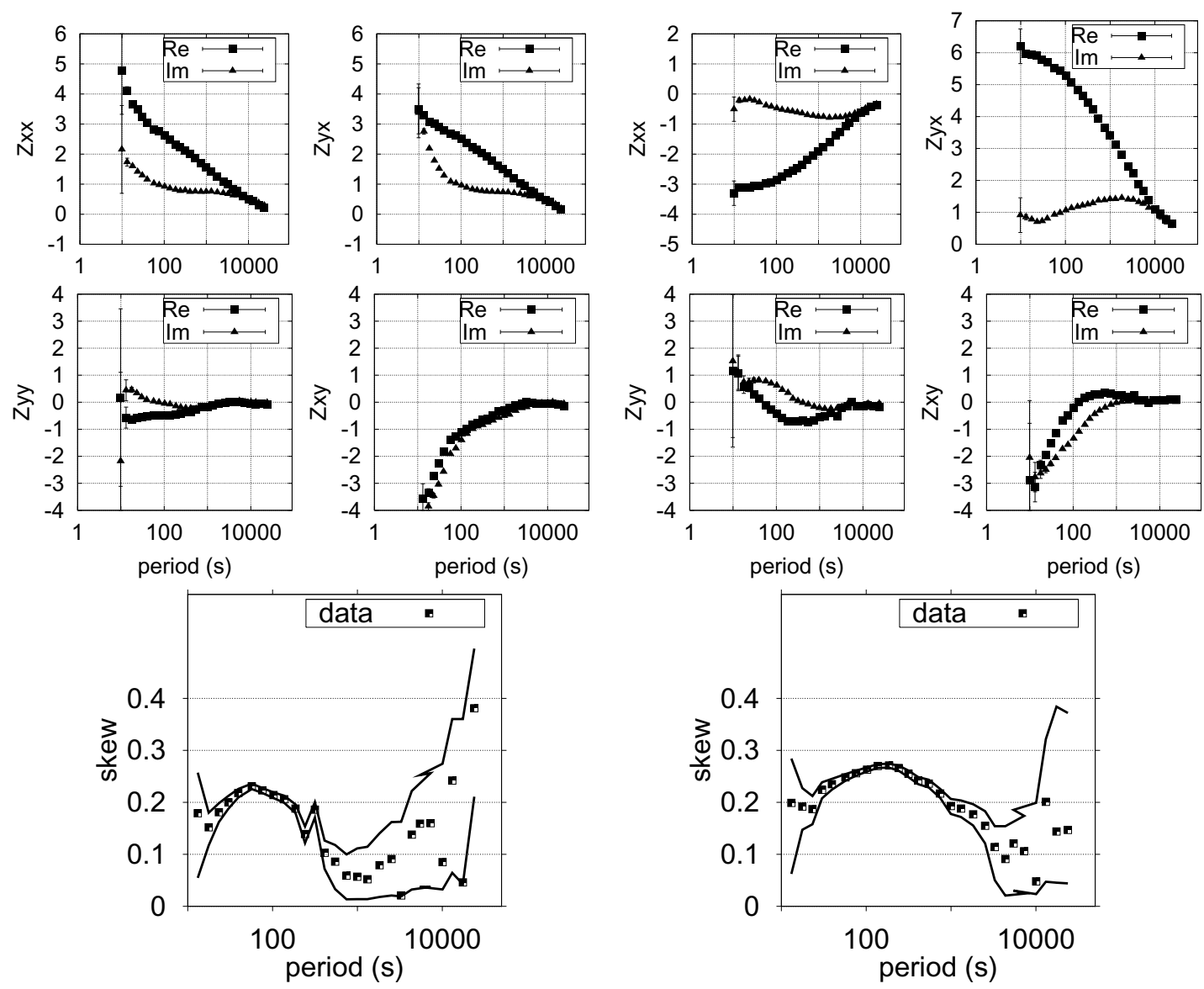

Fig. 6. Example with field data for site TIQ (left) and GER (right) located in the Southern Central Andes (SFB 267, 2001). Above: The tensor elements of the field data processed with a robust procedure (units in $\mathrm{km} / \mathrm{s}$ ). Below: The skew parameters of the data (dots) within the $95 \%$ confidence limits (lines).

example. The greatest uncertainties of skew (i.e., a broader confidence limit) are seen at the shortest and longest periods, where the relative errors of the tensor elements are the largest. At long periods, the smallest skew values close to the lower confidence limit could reflect a strong down bias from the true values, since the upper confidence limit is further higher.

The upper confidence limit for the skew can be used to analyse dimensionality, provided that the confidence limit value is not too large. For example, in this study area are cases where this upper threshold is far greater than 0.3 , while the lower confidence limit is near zero. Such values indicate an unreliable value for the skew due to large tensor elements error. As a result, further interpretation of dimensionality of the conductivity structure is inappropriate.

\section{Conclusions}

The derivation of the conditional probability function of the skew parameter allows estimation of a plausible confidence limit of the true value.

To analyse dimensionality on field data, it is advisable to treat the upper $95 \%$ confidence limit instead of the skew value itself. It was shown with synthetic data that the skew estimated from tensor elements scattered with $2 \%$ and $5 \%$ random Gaussian noise could suffer strong bias with regards to the true skew value. Where the confidence limit becomes extreme large $(\gg 0.3)$, the data should be discarded from the analysis.

The statistical procedure developed here can also be analogously applied for any other parameter which is a continuous and continuous differentiable non-linear function of the tensor elements.

Acknowledgments. I am thankful to Prof. Peter Weidelt for his initial and fundamental guidance in statistics. I acknowledge the constructive critic made by Dr. Richard Holme for improving the presentation of this manuscript. Thanks also to Dr. Yasuo Ogawa for the review. This study was funded by Deutscher Akademischer Austauschdienst (DAAD) within the framework of the Collaborative Research Project SFB 267 Deformation Processes in the Andes realized at the Freie Universität Berlin.

\section{Appendix A. Derivation of the Probability Func- tion of Skew for Normal Distributed Tensor Elements}

The conditional probability function (p.f.) of skew $G_{p}\left(\tilde{\eta}_{p}\right)$ derived from the transformation of variables (Eq. (9)) is:

$$
G_{p}\left(\tilde{\eta}_{p}\right)=P\left(-\frac{\tilde{\eta}_{p}^{2} d}{2}<\left(x_{p} s_{i} u_{i}+c\right)<\frac{\tilde{\eta}_{p}^{2} d}{2}\right)
$$

where $x_{p}$ is the conditional r.v. valid for the transformation of spaces (diagonal tensor element; Fig. 1), $u_{i}$ is the mean value of the variable $x_{i}, s_{i}$ and $c$ as defined in Eq. (8).

We make the variable transformation: 


$$
\begin{gathered}
y\left(x_{p}\right)=y_{p}=\left(x_{p} s_{i} u_{i}+c\right) \rightarrow x_{p}=\frac{y_{p}-c}{s_{i} u_{i}} \\
y_{p}=\frac{\eta_{p}^{2} d}{2}
\end{gathered}
$$

to treat the upper limit of p.f. written in Eq. (A.1a) in terms of the r.v. $x_{p}$. We refer to this as the p.f. $F\left(\tilde{y}\left(x_{p}\right)\right)$ :

$$
\begin{aligned}
F\left(\tilde{y}\left(x_{p}\right)\right) & =P\left(y_{p}<\frac{\tilde{\eta}_{p}^{2} d}{2}=\tilde{y}_{p}\right) \\
& =P\left(x_{p}<\frac{\tilde{y}_{p}-c}{s_{i} u_{i}}=x_{p}\left(\tilde{\eta}_{p}\right)\right) .
\end{aligned}
$$

The transformation of variable from $y_{p}$ to $x_{p}$ is valid since they fulfill the required properties for a valid transformation of spaces. The p.f. $F$ (Eq. (A.2a)) is transformed to the space of $x_{p}$, thus $F$ can be determined given a known p.f. for $x_{p}$.

The r.v. $x_{p}$ is assumed normally distributed with d.f. $\phi\left(x_{p}\right)$, mean value $u_{p}$ and standard deviation $\sigma_{p}$. Considering Eq. (A.2a), the p.f. $F$ as function of $\tilde{y}\left(x_{p}\right)=\tilde{\eta}_{p}^{2} d / 2$ (Eqs. (A.1b), (A.1c)) takes the form:

$F\left(\frac{\tilde{\eta}_{p}^{2} d}{2}\right)=\left\{\begin{array}{cc}\int_{-\infty}^{x_{p}\left(\tilde{\eta}_{p}\right)} \phi\left(x_{p}\right) d x_{p}=\psi_{o}\left(\frac{x_{p}\left(\tilde{\eta}_{p}\right)-u_{p}}{\sigma_{p}}\right) \\ \text { if }\left(s_{i} u_{i}\right)>0 \\ \int_{x_{p}\left(\tilde{\eta}_{p}\right)}^{\infty} \phi\left(x_{p}\right) d x_{p}=1-\psi_{o}\left(\frac{x_{p}\left(\tilde{\eta}_{p}\right)-u_{p}}{\sigma_{p}}\right) \\ \text { if }\left(s_{i} u_{i}\right)<0\end{array}\right.$

where $\psi_{o}$ is a Gaussian distribution with unit variance and zero mean. The two relations in the right side of Eq. (A.2b) come from the first condition of a valid transformation of spaces, i.e., $y_{p}\left(=\eta_{p}^{2} d / 2\right)$ is monotonic in $x_{p}$. For example, if $s_{i} u_{i}<0$, the r.v.'s defined in Eq. (A.1b) approach $y_{p}\left(x_{p}^{b}\right)<y_{p}\left(x_{p}^{a}\right)$ if $x_{p}^{b}>x_{p}^{a}$. This implies reversing the integration limits in Eq. (A.2b).

The p.f. of $\eta$ written in Eq. (A.1a) corresponds to a folded distribution, which is related to the p.f. $F$ defined in Eq. (A.2b) by the form (e.g., Dudewicz and Mishira, 1988):

$$
\begin{aligned}
F\left(\frac{\eta_{p}^{2} d}{2}\right)-F\left(\frac{-\eta_{p}^{2} d}{2}\right) \\
=\Psi_{o}\left(\frac{\left(\frac{\eta_{p}^{2} d}{2 s_{i} u_{i}}-\frac{c}{s_{i} u_{i}}\right)-u_{p}}{\sigma_{p}}\right) \\
-\Psi_{o}\left(\frac{\left(\frac{-\eta_{p}^{2} d}{2 s_{i} u_{i}}-\frac{c}{s_{i} u_{i}}\right)-u_{p}}{\sigma_{p}}\right)
\end{aligned}
$$

after expressing $x_{p}$ in terms of $\eta_{p}=\sqrt{\frac{2\left|x_{p}\left(s_{i} u_{i}\right)+c\right|}{d}}$ (Eq. (8)). The right term is obtained after standardizing $F$, valid for $s_{i} u_{i}>0$.

With the variable transformations

$$
\begin{aligned}
& x_{p}^{+}(\eta)=\frac{\eta^{2} d}{2 s_{i} u_{i}}-\frac{c}{s_{i} u_{i}} \\
& x_{p}^{-}(\eta)=\frac{-\eta^{2} d}{2 s_{i} u_{i}}-\frac{c}{s_{i} u_{i}}
\end{aligned}
$$

a final expression is defined for the conditional p.f. of $\eta$, by introducing these variables (Eq. (A.4)) in Eq. (A.3):

$$
G_{p}(\eta)=\left\{\begin{array}{c}
\Psi_{o}\left(\frac{x_{p}^{+}(\eta)-u_{p}}{\sigma_{p}}\right)-\Psi_{o}\left(\frac{x_{p}^{-}(\eta)-u_{p}}{\sigma_{p}}\right) \\
\text { if } s_{i} u_{i}>0 \\
\Psi_{o}\left(\frac{x_{p}^{-}(\eta)-u_{p}}{\sigma_{p}}\right)-\Psi_{o}\left(\frac{x_{p}^{+}(\eta)-u_{p}}{\sigma_{p}}\right) \\
\text { if } s_{i} u_{i}<0 .
\end{array}\right.
$$

This p.f. is related to the standardized folded normal distribution function (e.g., Dudewicz and Mishira, 1988). The two relations on the right come from the monotonic condition for a valid transformation of spaces as mentioned above (Eq. (A.2b)).

\section{References}

Bahr, K., Geological noise in magnetotelluric data: a classification of distortion types, Phys. Earth Planet. Int., 66, 24-38, 1991.

Brandt, S., Datenanalyse, 651 pp., Wissenschaftsverlag, Mannheim, 1992.

Dudewicz, E. J. and S. N. Mishira, Modern Mathematical Statistics, 838 pp., John Wiley \& Sons, New York, 1988.

Egbert, G. D. and J. R. Booker, Robust estimation of geomagnetic transfer function, Geophys. J. R. astr. Soc., 87, 173-194, 1986.

Fisz, M., Wahrscheinlichkeitsrechnung und Mathematische Statistik, Deutscher Verlag der Wissenschaften, Berlin, 1976.

Mackie, R. and J. Booker, Documentation for mtd3fwd and d3-to-mt, User Documentation, GSY-USA, Inc., 2261 Market St., Suite 643, San Francisco, CA 94114, 1999.

Mackie, R. L., J. T. Smith, and T. Madden, Three-dimensional electromagnetic modeling using finite difference equations: The magnetotelluric example, Radio Sci., 29, 923-936, 1994.

SFB 267, Deformation Processes in the Andes, Interaction between endogenic and exogenic processes during subduction orogenesis, Freie Universität Berlin, Technische Universität Berlin, GeoForschungsZentrum Potsdam, Universität Potsdam, Report for the research period 19992001, pp. 239-268, Berlin/Potsdam, March, 2001.

P. Lezaeta (e-mail: plezaeta@whoi.edu) 